\title{
RESEARCH
}

Open Access

\section{Spatiotemporal analysis of drug-resistant TB patients registered in selected districts of Karnataka, South India: a cross-sectional study}

Basavaraj Poojar ${ }^{1 *}$, K. Ashok Shenoy ${ }^{1}$, Poonam R. Naik², Ashwin Kamath', Jaya Prasad Tripathy ${ }^{3}$, P. Prasanna Mithra4, Mukta N. Chowta', M. N. Badarudeen ${ }^{5}$, Narasimhaswamy Nagalakshmi ${ }^{6,7}$, Vivek Sharma ${ }^{8}$,

Amrita N. Shamanewadi ${ }^{9}$ and Pruthu Thekkur ${ }^{3}$

\begin{abstract}
Background: Tuberculosis (TB) depicts heterogeneous spatial patterns with geographical aggregation of TB cases due to either ongoing person-to-person transmission or reactivation of latent infection in a community sharing risk factor. In this regard, we aimed to assess the spatiotemporal aggregation of drug-resistant TB (DR-TB) patients notified to the national TB program (NTP) from 2015 to 2018 in selected districts of Karnataka, South India.

Methods: This was a cross-sectional study among DR-TB patients notified from Dakshina Kannada, Udupi, and Chikamagalur districts of the state of Karnataka. Clinico-demographic details were extracted from treatment cards. The registered addresses of the patients were geocoded (latitude and longitude) using Google Earth. Using the QGIS software, spot map, heat maps and grid maps $25 \mathrm{~km}^{2}$ with more than the expected count of DR-TB patients were constructed.
\end{abstract}

Results: Of the total 507 patients studied, 376 (74\%) were males and the mean (standard deviation) age of the study participants was 41.4 (13.9) years. From 2015 to 2018, the number of patients increased from 85 to 209 per year, the area of aggregation in square kilometers increased from 113.6 to 205.7, and the number of rectangular grids with more than the expected DR-TB patients (> 1) increased from 12 to 47.

Conclusions: The increase in the number of DR-TB patients, area of aggregation, and grids with more than the expected count is a cause for concern. The NTP can use routine programmatic data to develop maps to identify areas of aggregation of disease for targeted TB control activities.

Keywords: Spatial heterogeneity, Drug-resistant tuberculosis, GIS, Spatial analysis, Geographic mapping, Spatial epidemiology

\footnotetext{
* Correspondence: basavaraj.poojar@manipal.edu;

basavaraj.poojar@gmail.com

${ }^{1}$ Department of Pharmacology, Kasturba Medical College, Mangalore,

Manipal Academy of Higher Education (MAHE), Manipal, Karnataka, India

Full list of author information is available at the end of the article
}

(c) The Author(s). 2020 Open Access This article is licensed under a Creative Commons Attribution 4.0 International License, which permits use, sharing, adaptation, distribution and reproduction in any medium or format, as long as you give appropriate credit to the original author(s) and the source, provide a link to the Creative Commons licence, and indicate if changes were made. The images or other third party material in this article are included in the article's Creative Commons licence, unless indicated otherwise in a credit line to the material. If material is not included in the article's Creative Commons licence and your intended use is not permitted by statutory regulation or exceeds the permitted use, you will need to obtain permission directly from the copyright holder. To view a copy of this licence, visit http://creativecommons.org/licenses/by/4.0/. 


\section{Introduction}

Globally, tuberculosis (TB) remains a major public health problem of concern with an estimated 10 million incident TB patients and 1.3 million deaths due to TB in the year 2017. Multidrug-resistant tuberculosis (MDR-TB, TB resistant to isoniazid and rifampicin) and extensively drugresistant tuberculosis (XDR-TB, MDR-TB plus resistance to any fluoroquinolones and at least one second-line injectable drug) have emerged globally and pose a threat to TB control efforts. The World Health Organization (WHO) estimated about 330,000 incident multidrug-resistant TB or rifampicin-resistant TB (MDR/RR-TB) patients globally in the year 2017 [1]. However, only $54 \%$ of the estimated were notified to the national TB program (NTP). Thus, almost half of the estimated MDR/RR-TB patients went either undiagnosed or diagnosed but not reported [2].

India is one of the 30 high-burden TB countries and has a triple burden of TB, TB/HIV, and MDR-TB. In 2017, it was estimated that there were $65,000 \mathrm{MDR} / \mathrm{RR}-\mathrm{TB}$ patients in the country with a prevalence of $2.8 \%$ among new TB cases and $12 \%$ among previously treated TB cases. Similar to global trends, there is a substantial gap in the detection and treatment of MDR/RR-TB patients in India. Of the estimated, only about $40 \%$ of the MDR/RR-TB patients are identified and initiated on treatment [2]. As the majority of patients are missed, identifying geographical areas with a high incidence of disease and adopting active case finding in such areas could help to reduce detection gap among DR-TB patients.

TB depicts heterogeneous spatial patterns with localized aggregation of cases due to either ongoing personto-person transmission [3] or reactivation of latent infection in a community sharing risk factor. Biological (malnutrition, HIV infection, and age distribution) and social determinants (poverty and behavioral risk factors) of TB are aggregated in geographical areas and eventually can lead to aggregation of TB patients in such areas. The previous studies on geospatial patterns of MDR-TB in Peru [4], Moldova [5], and Georgia [6] have shown MDR-TB patients to be aggregated within geographical areas. If the DR-TB is characterized by areas of concentrated risk rather than spatially uniform risk, intensitfied control activities at areas with concentrated risk may be efficient than adopting blanket approach for control of the disease.

Though geospatial analysis, identifying areas with the aggregation of $\mathrm{TB}$ patients, and focused $\mathrm{TB}$ control activities in such areas may be theoretically beneficial, such explorations are scarce. As a first step, there is a need for assessing the geospatial distribution of drug-resistant $\mathrm{TB}$ locally to detect whether there is any geographical aggregation of DR$\mathrm{TB}$ patients using routine program data. However, the inherent difficulties in geotagging the location of the patient and technical challenges in handling such data have limited the use of geospatial epidemiology in TB control efforts. Hence, as a desk review, we explored the possibility of geocoding the address of DR-TB patients using Google Maps and constructing the spatial heterogeneity maps. We geocoded all DR-TB patients treated under the Revised National Tuberculosis Control Program (RNTCP) in three selected districts of Karnataka from 2015 to 2018 to depict the spatiotemporal pattern of these patients.

\section{Methodology \\ Study design}

It is a cross-sectional descriptive study using secondary data collected routinely by the RNTCP of India.

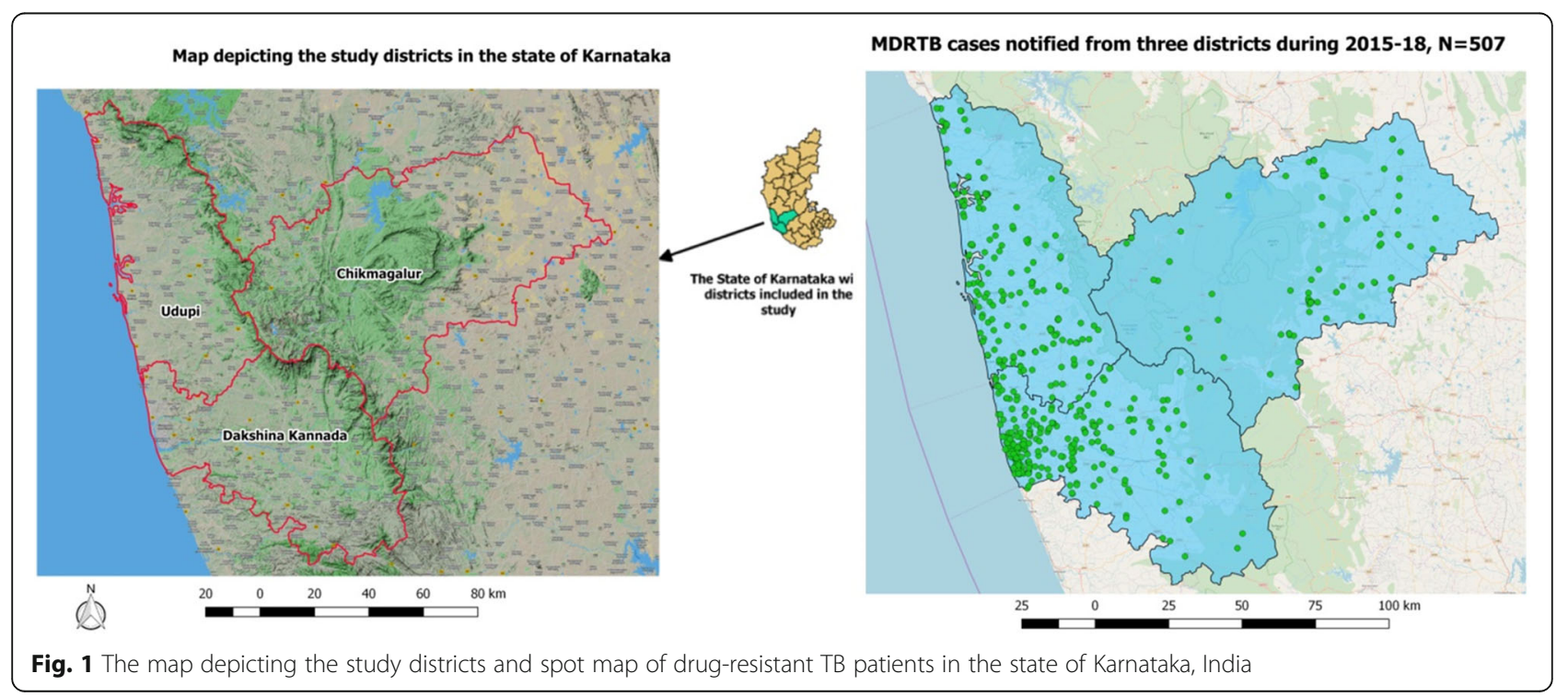




\section{Study setting}

\section{General setting}

Karnataka is a southern state of India. The approximate population of Karnataka is 66.8 million with a sex ratio of 973 females to 1000 males [7]. The RNTCP was universalized in the state by 2002 . In 2016, the state of Karnataka had an annual TB notification rate of 123/100, 000 individuals. Of the total cases notified, $79 \%(47,145)$ are new TB patients, and 29\% $(12,587)$ are previously treated. During the same year, the state had notified 1099, of which, 925 initiated DR-TB treatment [8].

\section{Specific setting}

Study site The study was conducted in the DR-TB center of Mangalore, Karnataka. The DR-TB center caters to all the DR-TB patients diagnosed in three districts, namely Dakshina Kannada, Chikmagalur, and Udupi (Fig. 1). According to the 2011 census [9], Dakshina Kannada district had a population of 2 million [10] and Chikmagalur and Udupi districts had a population of 1.1 million each $[11,12]$. The Revised National Tuberculosis Control Program (RNTCP) has 21 tuberculosis units (TUs) and 74 designated microscopy centers (DMCs) providing TB diagnosis and treatment support.

\section{Diagnosis of DR-TB}

The Programmatic Management of Drug-Resistant TB (PMDT) guideline was followed for the diagnosis and treatment of DR-TB in the study site. From 2015 to 2017 , the criteria-C was adopted for identifying presumptive MDR-TB patients [13]. All the presumptive MDR-TB patients were offered both phenotypic (culture and drug sensitivity) and molecular (Xpert MTB/RIF assay) drug susceptibility testing (DST) for detecting drug resistance to first-line drugs. From January 2018, universal drug susceptibility testing (UDST) was implemented in the study setting [8]. As per UDST, all the patients diagnosed with TB were provided upfront DST using Xpert MTB/Rif assay [14].

\section{DR-TB treatment initiation, follow-up, and treatment outcomes}

All the diagnosed DR-TB patients from the districts included in the study were referred to the DR-TB center at Mangalore for pretreatment evaluation and initiation of treatment. The patients reaching the DR-TB center were registered for care with a unique DR-TB registration number. The socio-demographic and clinical details, along with the address and phone number of the patient, were recorded in the DR-TB register by the staff nurse. Also, the PMDT treatment card was issued for each patient and had the same details recorded in it.
Table 1 Socio-demographic and clinical characteristics of DR-TB patients initiated on treatment under the national TB program in three selected districts of Karnataka during 2015 to 2018, N = 507

\begin{tabular}{|c|c|c|}
\hline Characteristics & Frequency & Percentage \\
\hline \multicolumn{3}{|l|}{ Age (in years) } \\
\hline $0-14$ & 2 & 0.3 \\
\hline $15-24$ & 51 & 10.0 \\
\hline $25-34$ & 123 & 24.2 \\
\hline $35-44$ & 116 & 22.8 \\
\hline $45-54$ & 110 & 21.6 \\
\hline $55-64$ & 64 & 12.6 \\
\hline$\geq 65$ & 36 & 7.1 \\
\hline Not recorded & 5 & 0.9 \\
\hline \multicolumn{3}{|l|}{ Gender } \\
\hline Male & 377 & 74.3 \\
\hline Female & 129 & 25.4 \\
\hline Not recorded & 1 & 0.1 \\
\hline \multicolumn{3}{|l|}{ District } \\
\hline Dakshina Kannada & 283 & 55.8 \\
\hline Udupi & 151 & 29.7 \\
\hline Chikamagalur & 73 & 14.3 \\
\hline \multicolumn{3}{|l|}{ Year (total number of cases) } \\
\hline 2015 & 85 & 16.7 \\
\hline 2016 & 99 & 19.5 \\
\hline 2017 & 114 & 22.4 \\
\hline 2018 & 209 & 41.2 \\
\hline \multicolumn{3}{|l|}{ Type of TB } \\
\hline New & 340 & 67.0 \\
\hline Retreatment after loss to follow-up & 43 & 8.4 \\
\hline Retreatment after failure & 55 & 10.8 \\
\hline Retreatment/relapse & 69 & 13.6 \\
\hline \multicolumn{3}{|l|}{ DST used for diagnosis } \\
\hline CDST & 30 & 5.9 \\
\hline Xpert MTB & 260 & 51.2 \\
\hline LPA & 216 & 42.6 \\
\hline Not recorded & 1 & 0.1 \\
\hline \multicolumn{3}{|l|}{ HIV status } \\
\hline Positive & 18 & 3.5 \\
\hline Negative & 346 & 68.2 \\
\hline Unknown & 4 & 0.7 \\
\hline Not recorded & 139 & 27.4 \\
\hline \multicolumn{3}{|l|}{ ART status $(N=18)$} \\
\hline On ART & 17 & 94.4 \\
\hline Not on ART & 1 & 5.6 \\
\hline
\end{tabular}

Abbreviation: DR-TB drug-resistant tuberculosis, DST drug sensitivity testing, CDST culture and drug susceptibility test, LPA line probe assay, MTB Mycobacterium tuberculosis, Rif rifampicin, HIV human immunodeficiency virus *Column percentage 
At the DR-TB center, the patients were hospitalized for pretreatment evaluation and treatment initiation. All DR-TB patients were prescribed drugs based on PMDT guidelines. After initiating the therapy, patients were monitored at the DR-TB center for 1 to 2 weeks. Once a patient is stable and tolerating the second-line drugs, then he/she was referred to the peripheral health institute (PHI) nearest to patient residence for further care and monitoring with ambulatory DOTS-Plus treatment at PHI. All the details of the treatment course in the DR-TB center and referral details were updated in the DR-TB treatment card and DR-TB register.

\section{Diagnosis of DR-TB and treatment by private providers}

The DR-TB patients diagnosed by private providers are notified to RNTCP. As the treatment of DR-TB is complicated, majority of the patients are referred to public health facilities for treatment and eventually initiated treatment at the DR-TB center. However, a few patients might continue to be treated by private providers.

\section{Study population}

All DR-TB patients initiated on TB treatment from a DR-TB center at Mangalore from January 2015 to December 2018 were included.

\section{Data variables, sources of data, and data collection}

Data on socio-demographic and clinical characteristics and drug-resistant pattern like PMDT number, age, gender (male/female/others), village/ward, taluk, district, name of TU, name of PHI, DOTS provider, date of DST, date of DST results, date of registration, type of TB (new/treatment after loss to follow-up/treatment after failure/recurrent/relapse), site of TB (pulmonary/extra-pulmonary), type of DST used for diagnosis (CDST/Xpert MTB/RIF assay), molecular test results (not done/Mtb+/Rif $+/ \mathrm{Mtb}+/ \mathrm{Rif}-/ \mathrm{Mtb}$ $-/ \mathrm{MTB}+$ Rif indeterminate), type of DR-TB (RR-TB/
MDR-TB/XDR-TB), resistance to isoniazid (yes/no/ not available), resistance to ethambutol (yes/no/not available), resistance to pyrazinamide (yes/no/not available), resistance to streptomycin (yes/no/not available), resistance to fluoroquinolones (yes/no/not available), resistance to kanamycin (yes/no/not available), HIV status (yes/no/not available), ART status (yes/no/not available/not applicable), weight at initiation of treatment, tobacco use (yes/no/not available), and alcohol use (yes/no/not available) were extracted from DR-TB treatment card and DR-TB register maintained at DR-TB center.

\section{Geocoding}

The geocode (latitude and longitude) of each patient was obtained based on the registered address using Google Earth pro version 7.3.2.5776 application. The village/ ward/street of the patient was used to geocode the address [15-17]. The details like taluk, district, and PHI were used to locate the village/ward/street on the geospatial map. In case of non-availability of the village/ ward/street, the support was sought from Senior Treatment Supervisor (STS) of the TU or PHI to which the patient was referred for DOTS-Plus. If the village/ward/ street provided in the address is not found on Google Maps, the nearest village as suggested by the STS or staff of PHI was geocoded. The geocodes were noted in decimal degrees format with a minimum of five digits after the decimal point [18].

\section{Data entry and analysis}

Data were double entered and validated using EpiData Entry software (EpiData Association, Odense, Denmark) and analyzed using EpiData analysis (version 2.2.2.182, EpiData Association, Odense, Denmark) and Stata version 12.0 (STATA Corp., College, TX, USA). Sociodemographic and clinical characteristics and drug-

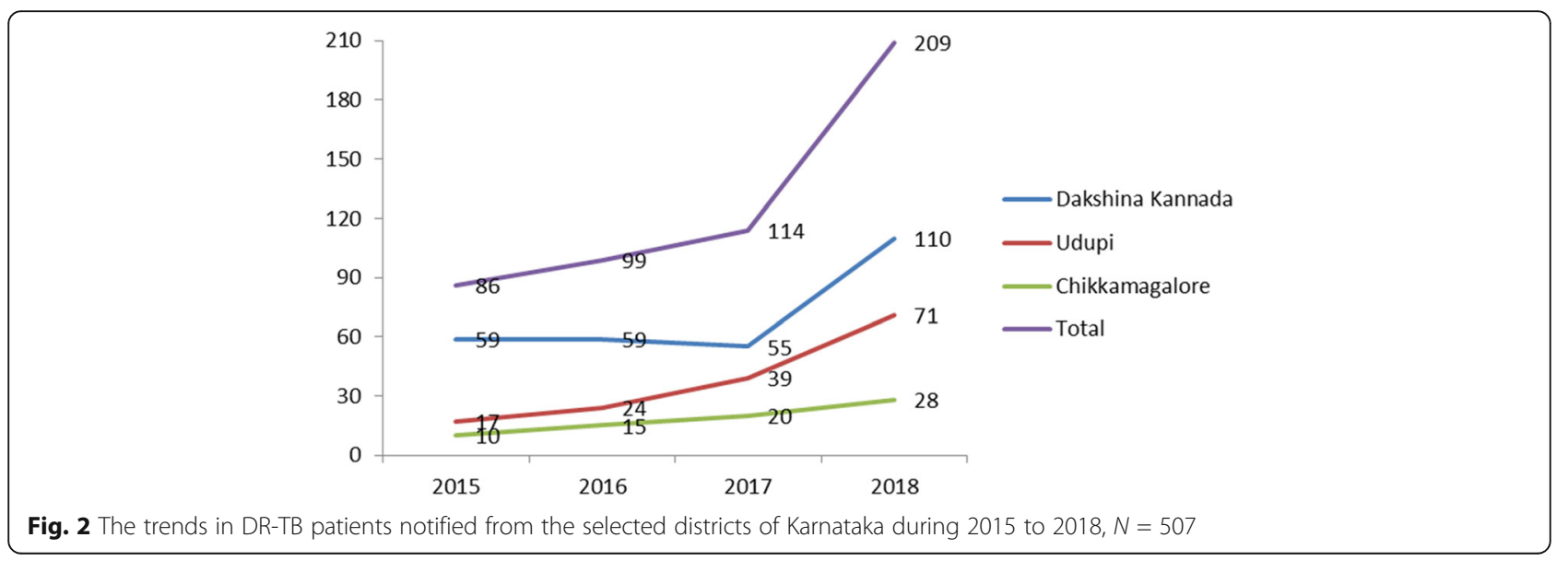


resistant patterns were summarized using numbers and percentages.

The QGIS version 2.18.15 (QGIS Developer team, Las Palmas (2016)) was used to plot the drug-resistant TB patients, and a map of geospatial distribution was constructed. The heat maps depicting spatial heterogeneity [19] were constructed for the year 2015 to 2018. The quadratic weight was used as a density measure [20], and the heat maps $[5,20]$ were constructed with a buffer radius appropriate to the layer units [21]. The heat map constructed depicts the geographical aggregation of the DR-TB patients but not the relatedness of the cases.

The grid maps were constructed with each grid of 25 $\mathrm{km}^{2}$. Within each grid, the number of DR-TB cases was counted. Those cases with more than the expected number of DR-TB cases were colored red. The expected DRTB patients per grid were calculated with a population density of 282 per square kilometer in three districts, and the estimated number of TB patients per $25 \mathrm{~km}^{2}$ is 15 (considering estimated annual TB incidence of 210/1, 000,000 in the country) [14]. The expected number of DR-TB is 1 per $25 \mathrm{~km}^{2}$ assuming the proportion of DRTB cases among TB patients to be $6 \%$.

\section{Results}

In total, 507 DR-TB patients were notified and initiated on treatment from 2015 to 2018 in the study districts. The mean (SD) age of the study participants was 41.4

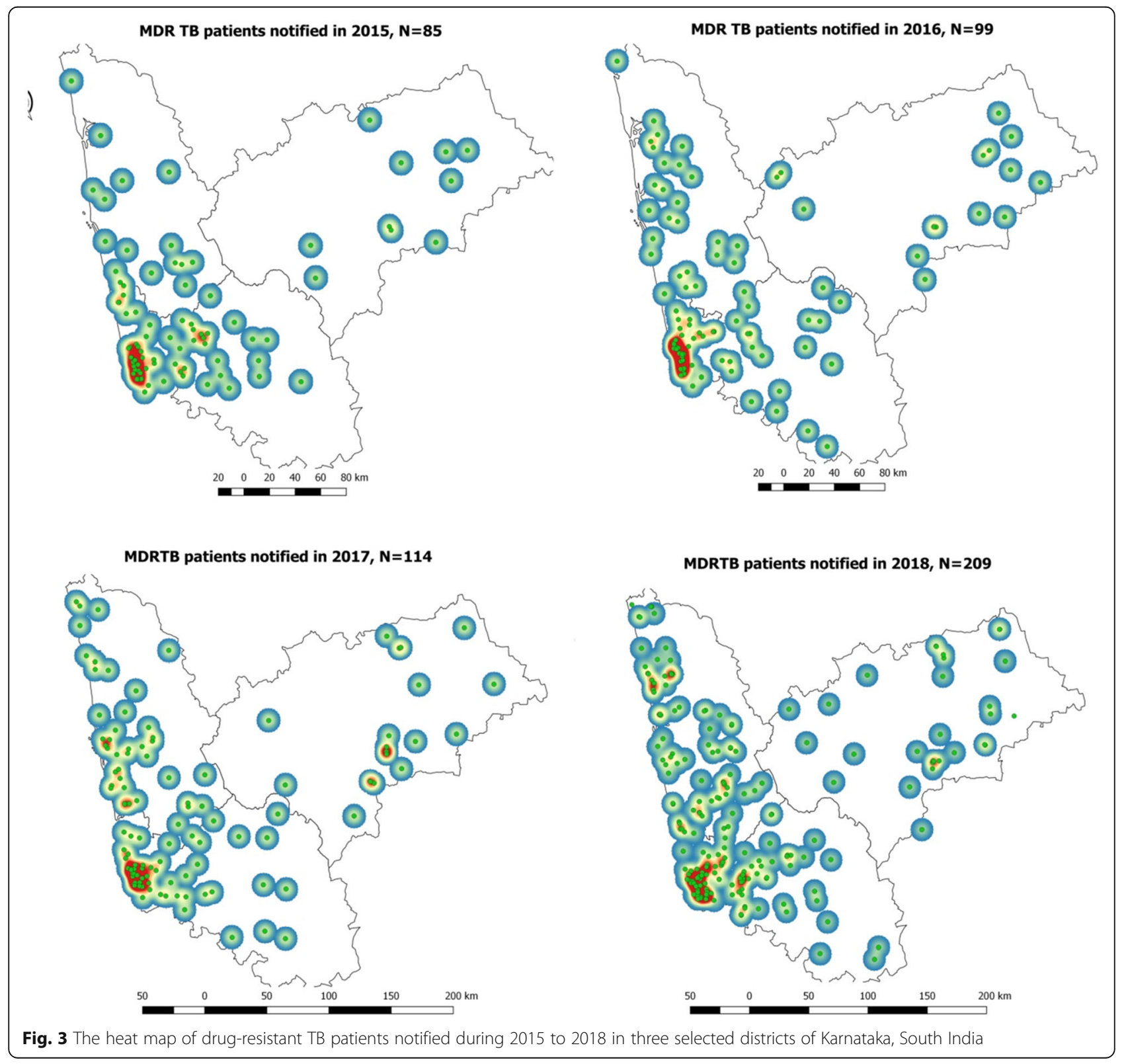


(13.9), and $376(74 \%)$ were males. Of the total, about 452 (89\%) of the DR-TB patients were new TB patients. The DR-TB patient characteristics included in the study are shown in Table 1. During the study period, the number of DR-TB cases increased from 85 in the year 2015 to 209 in 2018. Figure 2 depicts the trends in notified DR-TB patients across the study districts.

Figure 1 shows the spot map of DR-TB patients, and Fig. 3 depicts the year-wise "heat maps" generated for 2015 to 2018. The red color in the "heat map" indicates the area with the aggregation of DR-TB cases. The sum of the area of aggregation in square kilometers was 113.6, 95.2, 131.4, and 205.7 during 2015, 2016, 2017, and 2018, respectively. The number of rectangular grids of $25 \mathrm{~km}^{2}$ with more than the expected DR-TB patients
(> 1) increased from 12 to 47 during the year 2015 to 2018 (Fig. 4).

Figure 5 and Table 2 show the geospatial and frequency distribution of resistance patterns of DR-TB patients, respectively. About $38 \%$ and $12 \%$ of the DR-TB patients had resistance to isoniazid and fluoroquinolones, respectively. The geospatial distribution did not depict the localized aggregation of similar resistance pattern. About $10(2 \%)$ of the DR-TB patients had XDRTB.

\section{Discussion}

This is the first study from India assessing the spatiotemporal trends of DR-TB patients using geocoded data of individual patients. The study showed spatial

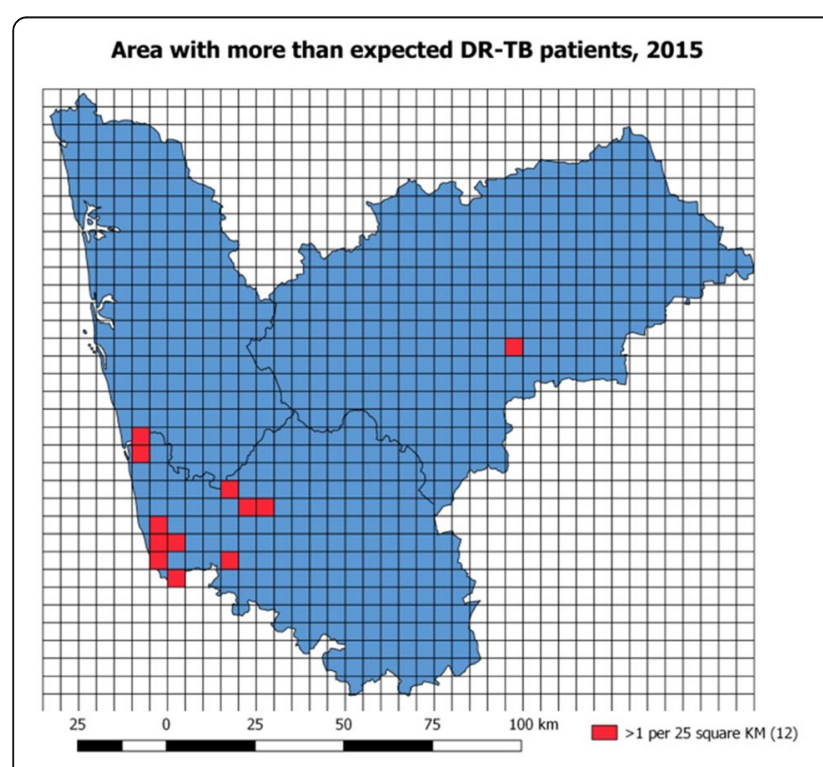

Area with more than expected DR-TB patients, 2017

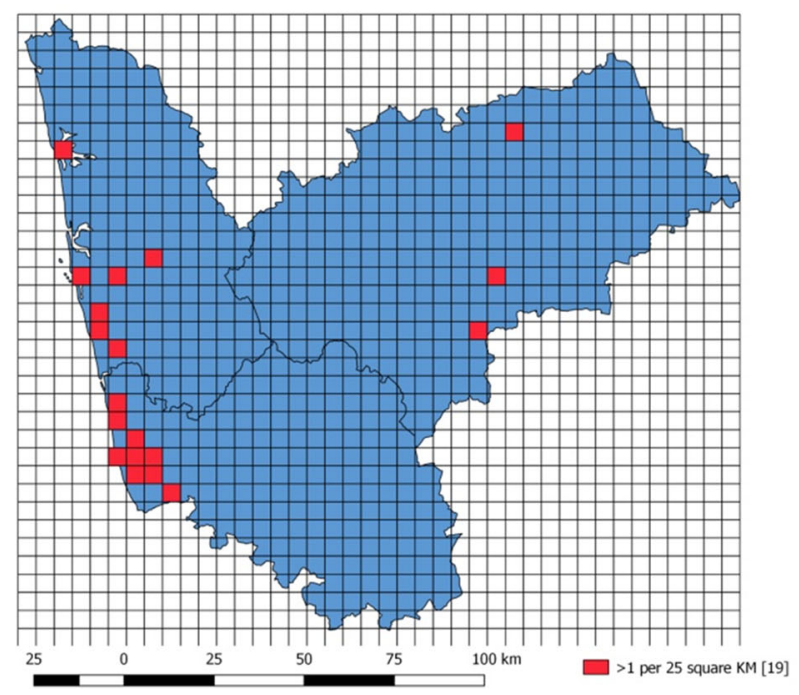

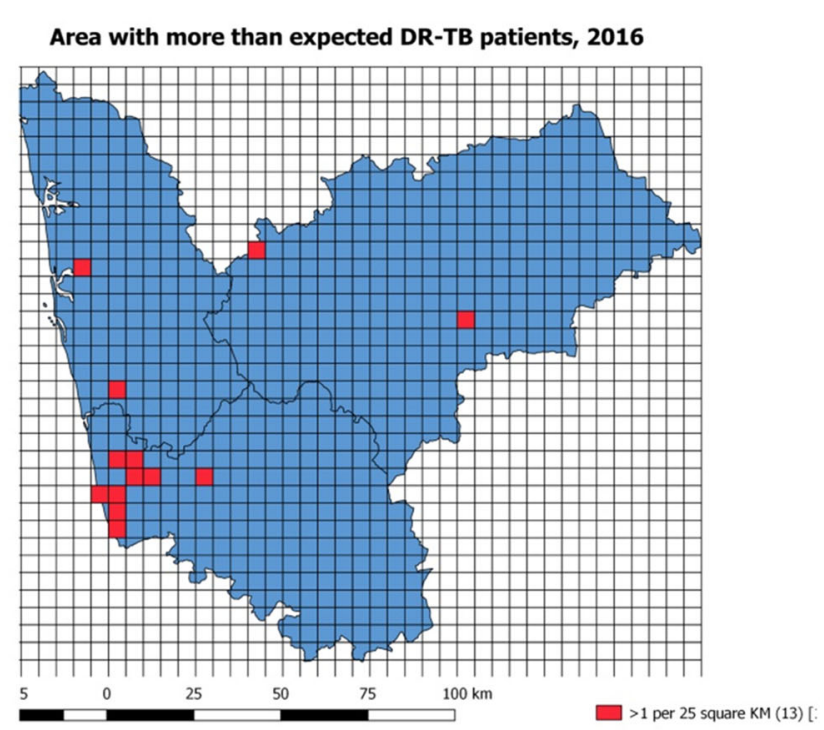

Area with more than expected DR-TB patients, 2018

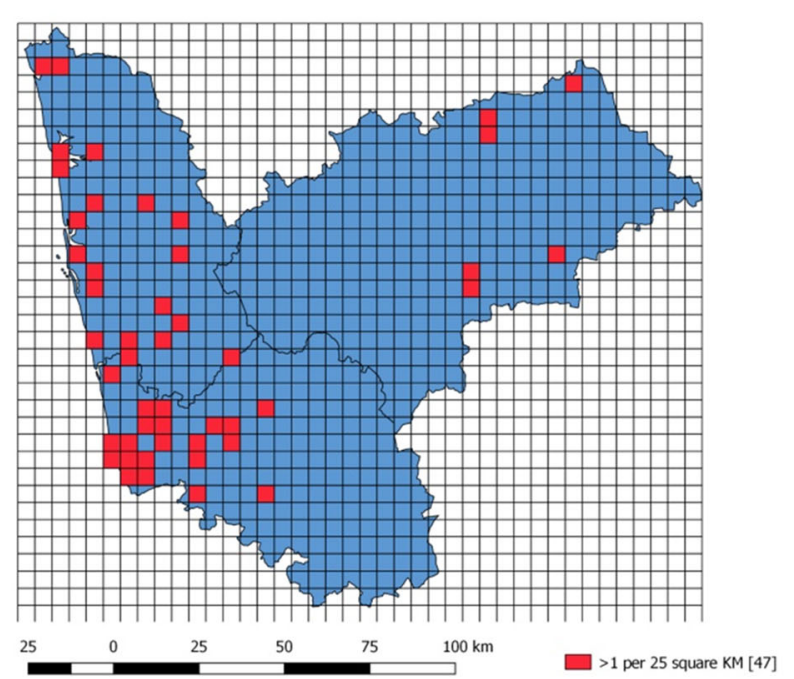

Fig. 4 Map depicting the grids $\left(25 \mathrm{~km}^{2}\right)$ with more than the expected number of DR-TB patients 


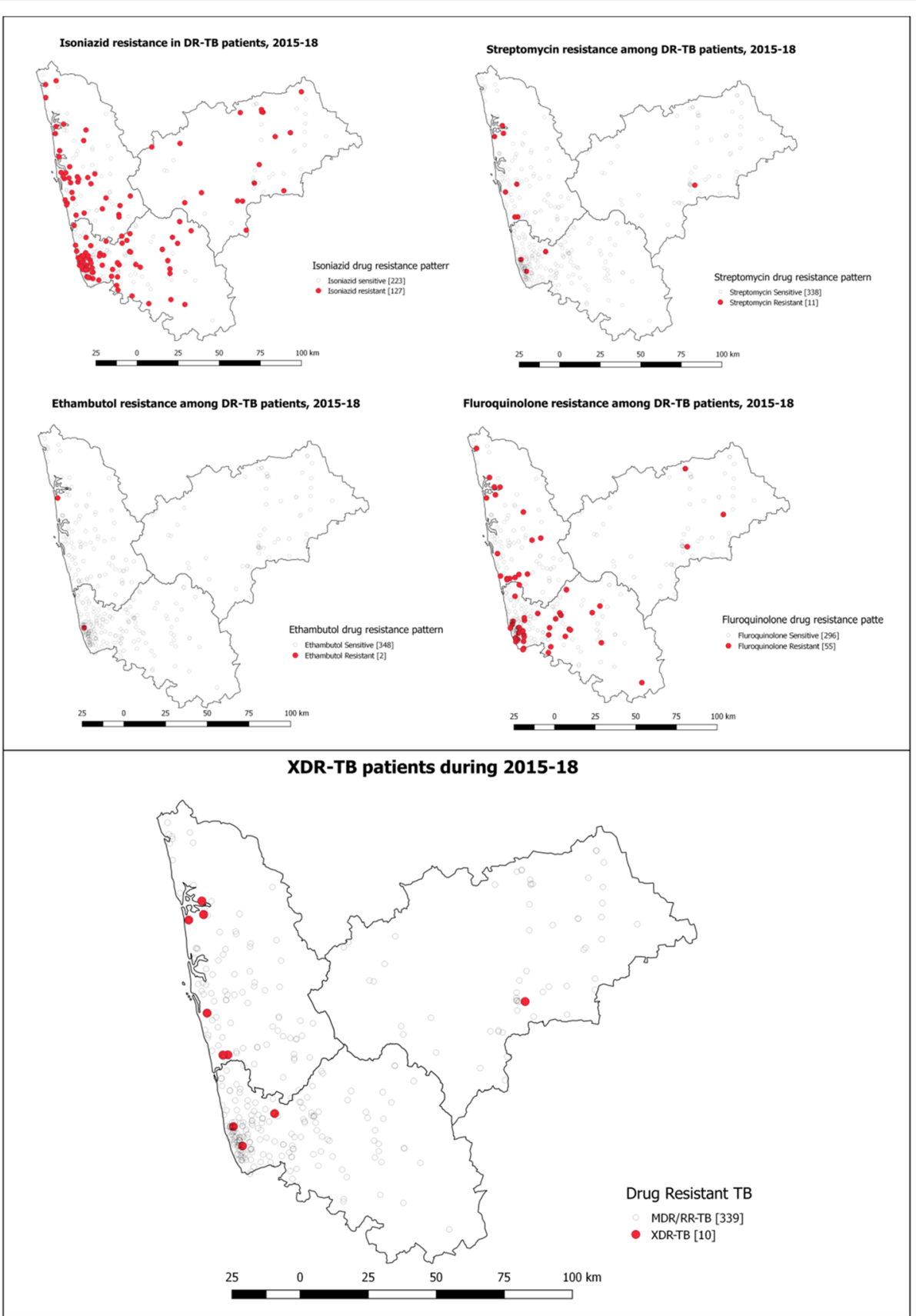

Fig. 5 Spatial pattern of drug-resistant pattern of DR-TB patients notified during 2015 to 2018 in three selected districts of Karnataka, South India

heterogeneity with an aggregation of DR-TB patients. Over the years (2015-2018), there was an increase in the number of DR-TB patients, the sum of the area of aggregation of patients, and the number of grid units with more than the expected DR-TB patients in the study districts. About one in ten DR-TB patients had resistance to fluoroquinolone.

There was an increase in the number of DR-TB patients in the study districts. The increasing trend was noted in each of the study districts. The annual TB reports have also reported an increase in notified DR-TB patients from 2015 to 2017. The increase might be either due to improved care delivery and adherence to protocols for the detection of DR-TB or due to the uninterrupted transmission of DR-TB. The massive increase in the number of DR-TB cases in 2018 could be due to introduction of the universal DST (DST made available for all $\mathrm{TB}$ patients). Improving the diagnostic services 
Table 2 Drug sensitivity pattern of DR-TB patients initiated on treatment under the national TB program in three selected districts of Karnataka during 2015 to 2018, N = 507

\begin{tabular}{lll}
\hline Drug sensitivity test & Frequency & Percentage \\
\hline Resistance to isoniazid & 195 & 38.4 \\
Yes & 292 & 57.6 \\
No & 20 & 4.0 \\
Not available & & \\
Resistance to ethambutol & 2 & 0.3 \\
Yes & 485 & 95.6 \\
No & 20 & 4.0 \\
Not available & & \\
Resistance to streptomycin & 12 & 2.3 \\
Yes & 474 & 93.4 \\
No & 21 & 4.1 \\
Not available & & \\
Resistance to fluoroquinolones & & 11.6 \\
Yes & 59 & 84.6 \\
No & 429 & 3.7 \\
Not available & 19 & 1.9 \\
XDR-TB & & 93.8 \\
Yes & 10 & 4.1 \\
No & 476 & \\
Not evaluated & 21 & \\
\hline Abrevaton DR-TB drugrestant tubeusis & & \\
\hline
\end{tabular}

Abbreviation: $D R-T B$ drug-resistant tuberculosis

and making the services accessible might have increased the number of notified DR-TB cases. The other potential reasons for the increase in notification of DR-TB patients are imporved quality of DR-TB in public sector and also the disinterest of private providers in treating DR-TB patients within the private sector.

The previous studies in Peru [4] and Moldova [5] have reported spatial aggregation of DR-TB patients. Similar findings were seen in the current study. However, the study results are not comparable as the methods used in generating the spatial heterogeneity maps were different. The increase in the area of aggregation suggests there was a creation of local foci, which could be due to potential internal transmission. None of the previous studies explored the spatiotemporal aggregation.

The study has a few strengths. First, geocoding was done for all the notified cases during the study reference period as all the addresses could be extracted and traced back. Hence, there was no selection bias due to missing information in routine programmatic records. Second, the individual DR-TB patients were geocoded. This limited the aggregation bias of constructing heat maps using administrative boundaries and provided the opportunity to construct the heat maps based on the occurrence of an event. Third, the sample size was good for conducting spatial heterogeneity analysis and also spatiotemporal analysis. Fourth, double data entry and validation were used. This helped to limit the data entry errors and improved quality of data, more so with variables like latitude and longitude with five digits after the decimal point.

The study has a few limitations. First, the geocodes were approximated to village/ward or street level and thus failed to geotag the house of the patient. However, the villages in this part of the country have a small area [22] and therefore might not have led to gross imprecision in marking the DR-TB patients [22]. Second, the expected number of cases per $25 \mathrm{~km}^{2}$ was calculated based on the estimated TB incidence rate for India and averaging the population density in the three districts. This might have reduced the internal validity of the study findings. However, the incidence rate in the study districts might be lower than that of the country estimates, and thus, the expected number of DR-TB patients is an overestimate. The population across the district is not uniform, and there might be areas with a higher population density with a relatively higher number of expected DR-TB patients leading to underestimation. Thus, on the whole, the estimation of expected DR-TB patients per $25 \mathrm{~km}^{2}$ might be close to a real-world scenario. Third, there were no village- and ward-level shapefiles, which might have helped us to calculate the rates of occurrence of the DR-TB. Hence, we had to calculate the expected number of DR-TB patients, which might be inferior to rates. Fourth, the data series was available only for 4 years and had extreme variation. This restricted the use of time series analysis and prediction models. Fifth, we might have missed the undetected and not notified DR-TB patients. This could have limited the validity of heat maps as the not notified and undetected case might contribute to disease transmission but not accounted for in the analysis. Sixth, the study might have failed to capture the natural trend in the occurrence of the DR-TB as there was a change in the diagnostic algorithm, which could have influenced the case detection in the year 2018.

Despite several limitations, the study has a few implications. First, over the years, there was an increase in the number of cases and also in the area of aggregation of cases. The RNTCP needs to explore the potential reasons for this increase in DR-TB patients. If the programmatic change of upfront DST has brought this change, then this is a promising step in efforts towards control of DR-TB in India. However, if the increase is due to the transmission of the disease, then this is a cause for concern. Second, the geocoding and generation of heat maps provided insights on the aggregation of disease 
and the spread of disease to new areas over the years. The program can train the data entry operators to geocode the villages of DR-TB patients and generate maps to help in decision-making. Third, the utility of concentrated TB control efforts in areas with DR-TB aggregation on reducing the transmission and burden of DR-TB needs to be explored. Fourth, on moving towards the elimination of TB, there is a need for developing the geospatial monitoring indicators like "number of TB or DR-TB case per square kilometer" area and prioritize areas with high number of cases for intensified TB control activities.

\section{Conclusions}

The increase in the number of DR-TB patients, area of aggregation, and grids with more than the expected count is a cause for concern. The NTP can use routine programmatic data to develop maps to generate local foci for targeted TB control activities.

\section{Abbreviations}

CDST: Culture and drug susceptibility test; DMC: Designated microscopy center; DOTS: Directly observed treatment, short-course; DR-TB: Drugresistant TB; DST: Drug susceptibility testing; HIV: Human immunodeficiency virus; LPA: Line probe assay; MDR-TB: Multidrug-resistant tuberculosis; MTB: Mycobacterium tuberculosis; NTP: National TB program; PHI: Peripheral health institute; PMDT: Programmatic Management of Drug-Resistant TB; Rif: Rifampicin; RNTCP: Revised National Tuberculosis Control Program; TB: Tuberculosis; TU: Tuberculosis unit; UDST: Universal drug susceptibility testing; WHO: World Health Organization; XRD-TB: Extensively drug-resistant tuberculosis

\section{Acknowledgements}

This research was conducted as a part of the "National Operational Research Training Course 2018-19" organized by Project Axshya, funded by The Global Fund, and implemented by The International Union Against Tuberculosis and Lung Diseases (The Union), South-East Asia Regional Office, New Delhi, India. The training course was conducted in collaboration with Revised National Tuberculosis Control Program, Ministry of Health and Family Welfare, Government of India and National Institute for TB and Respiratory Diseases, New Delhi, India.

The training is based on "The Union/Medécins sans Frontières (MSF)" model OR course and which has been adapted as SORT IT (Structured Operational Research and Training Initiative) coordinated by TDR, the Special Programme for Research and Training in Tropical Diseases at the World Health Organization Mentorship, and facilitation for this course was provided through The Union South-East Asia Office, New Delhi; the Centre for Operational Research, The Union, Paris, France; Baroda Medical College, Vadodara; Médecins Sans Frontières, New Delhi; ESIC Medical College and PGIMSR, Bengaluru; North Delhi Municipal Corporation Medical College, Hindu Rao Hospital, New Delhi; GMERS Medical College, Vadodara; Postgraduate Institute of Medical Education and Research, Chandigarh, India; and Yenepoya Medical College, Mangalore.

\section{Authors' contributions}

$\mathrm{BP}$ was the principal investigator and was involved in the conception, designing of the protocol, development of data collection tool, data analysis, and preparation of the first draft of the article. PRN and JPT were SORT IT course mentors involved in preparing the protocol, interpreting the data, and preparing the first draft. AK, JPB, MNC, and BMN were local mentors and provided support in the conception, designing of the protocol, and acquisition of data. PMP, NN, VS, and ANS were involved in designing the protocol and acquisition of data. PT was a SORT IT mentor and senior author involved in the conception, designing of the protocol, development of data collection tool, data analysis, and preparation of the first draft of the article. All the authors reviewed and approved the final version of the manuscript.

\section{Funding}

The training course under which this research was conducted was funded by The Global Fund to Fight AIDS, Tuberculosis and Malaria (GFATM). The funders had no role in the study design, data collection and analysis, decision to publish, or preparation of the manuscript.

\section{Availability of data and materials}

The datasets used and/or analyzed during the current study are available from the corresponding author on reasonable request.

\section{Ethics approval and consent to participate}

The study was approved by the Ethics Advisory Group of the International Union Against Tuberculosis and Lung Disease, Paris, France (EAG number: 83/18). The local ethics committee approval was obtained from the ethics committee of Kasturba Medical College (IEC KMC MLR 09-18/304). Permission was sought from the District TB Officer of the Dakshina Kannada district to access DR-TB treatment cards and DR-TB register maintained at the DR-TB center of Mangalore. As this was a retrospective cohort study without direct patient interaction, the ethics committees provided a waiver from informed consent from the study participants.

\section{Consent for publication}

As this was a retrospective cohort study without direct patient interaction, the ethics committees provided a waiver from informed consent from the study participants. All the authors approved the final draft of manuscript and consented to be co-authors.

\section{Competing interests}

The authors declare that they have no competing interests.

\section{Author details}

${ }^{1}$ Department of Pharmacology, Kasturba Medical College, Mangalore, Manipal Academy of Higher Education (MAHE), Manipal, Karnataka, India. ${ }^{2}$ Department of Community Medicine, Yenepoya Medical College, Mangalore, Yenepoya (Deemed to be University), Mangalore, Karnataka, India. ${ }^{3}$ Centre for Operational Research, International Union Against Tuberculosis and Lung Disease, Paris, France. ${ }^{4}$ Department of Community Medicine, Kasturba Medical College, Mangalore, Manipal Academy of Higher Education (MAHE), Manipal, Karnataka, India. ${ }^{5}$ District TB Center, Mangalore, India. ${ }^{6}$ Department of Microbiology, Melaka Manipal Medical College, Manipal Academy of Higher Education, Manipal, India. ${ }^{7}$ Department of Microbiology and Immunology, College of Medicine, American University of Antigua, St John's, Antigua and Barbuda. ${ }^{8}$ Tuberculosis Health Action and Learning Initiative (THALI), JSI India, New Delhi, West Bengal, India. ${ }^{9}$ Department of Community Medicine, MVJ Medical College and Research Hospital (MVI\&MCRH) Hoskote, Bangalore, India.

Received: 31 July 2019 Accepted: 27 February 2020

Published online: 10 March 2020

\section{References}

1. Tuberculosis. [cited 2019 May 19]. Available from: https:/www.who.int/ news-room/fact-sheets/detail/tuberculosis.

2. World Health Organization. Global tuberculosis [Internet]. Vol. 312, Jama. 2017. 28-58 p. Available from: http://www.who.int/tb/publications/global_ report/MainText_13Nov2017.pdf?ua=1.

3. Yates TA, Khan PY, Knight GM, Taylor JG, McHugh TD, Lipman M, et al. The transmission of Mycobacterium tuberculosis in high burden settings. Lancet Infect Dis [Internet]. 2016;16(2):227-38.

4. Manjourides J, Lin HH, Shin S, Jeffery C, Contreras C, Cruz JS, et al. Identifying multidrug resistant tuberculosis transmission hotspots using routinely collected data. Tuberculosis. 2012;92(3):273-9.

5. Theron G, Jenkins HE, Cobelens F, Abubakar I, Khan AJ, Cohen T, Dowdy DW. Data for action: collection and use of local data to end tuberculosis. Lancet. 2015;386(10010):2324-33.

6. Alene KA, Viney K, McBryde ES, Clements ACA. Spatial patterns of multidrug resistant tuberculosis and relationships to socioeconomic, 
demographic and household factors in northwest Ethiopia. PLoS One. 2017;12(2):1-14.

7. Population of Karnataka 2018 - Karnataka population, sex ratio, literacy rate [Internet]. [cited 2018 Aug 23]. Available from: http://www.indiaonlinepages. com/population/karnataka-population.html.

8. ndcp_cd_rntcp [Internet]. [cited 2018 Aug 23]. Available from: https://www. karnataka.gov.in/hfw/nhm/pages/ndcp_cd_rntcp.aspx.

9. Manley D, Flowerdew R, Steel D. Scales, levels and processes: studying spatial patterns of British census variables. Comput Environ Urban Syst. 2006:30(2):143-60.

10. Dakshina Kannada District : Census 2011 data. Census 2011; [cited 2018 Aug 22]. Available from: http://www.census2011.co.in/census/district/252dakshina-kannada.html.

11. About Chikkamagaluru. www.chickmagalur.nic.in [Internet]. [cited 2018 Aug 22]; Available from: http://www.chickmagalur.nic.in/htmls/about_ chickmagalur.htm.

12. Census of India 2001: Data from the 2001 Census, including cities, villages and towns (Provisional) [Internet]. Census Commission of India; [cited 2018 Aug 22]. Available from: http://www.censusindia.net/results/town.php?stad= A\&state5=999.

13. TOG-Chapter 4-Treatment of TB :: Central TB Division [Internet]. [cited 2019 Jul 29]. Available from: https://tbcindia.gov.in/index1.php?sublinkid=4611 \&level $=3 \&$ lid $=3221 \&$ lang $=1$

14. Central TB Division. Technical and operational guidelines for tuberculosis control in India [Internet]. New Delhi, India; 2016. Available from: https:// www.tbcindia.gov.in/index1.php?lang=1\&level=2\&sublinkid=4573\&lid $=3177$.

15. Kirby RS, Delmelle E, Eberth JM. Advances in spatial epidemiology and geographic information systems. Ann Epidemiol. 2017;27(1):1-9.

16. Jacquez GM. A research agenda: does geocoding positional error matter in health GIS studies? Spatial Spatio-Temporal Epidemiol. 2012;3(1):7-16.

17. Zandbergen PA. A comparison of address point, parcel and street geocoding techniques. Comput Environ Urban Syst. 2008;32(3):214-32.

18. Lai PC, So FM, Chan KW. Spatial epidemiological approaches in disease mapping and analysis. CRC press; 2008.

19. Netek R, Pour T, Slezakova R. Implementation of heat maps in geographical information system - exploratory study on traffic accident data. Open Geosci. 2018:10(1):367-84.

20. Shaweno D, Karmakar M, Alene KA, Ragonnet R, Clements AC, Trauer JM, et al. Methods used in the spatial analysis of tuberculosis epidemiology: a systematic review. BMC Med. 2018;16(1):1-18.

21. QGIS Heatmap Using Kernel Density Estimation Explained [Internet]. [cited 2019 Sep 28]. Available from: https://www.geodose.com/2017/11/qgisheatmap-using-kernel-density.html.

22. Dwyer-Lindgren L, Cork MA, Sligar A, Steuben KM, Wilson KF, Provost NR, Mayala BK, VanderHeide JD, Collison ML, Hall JB, Biehl MH. Mapping HIV prevalence in sub-Saharan Africa between 2000 and 2017. Nature. 2019; 570(7760):189.

\section{Publisher's Note}

Springer Nature remains neutral with regard to jurisdictional claims in published maps and institutional affiliations.

Ready to submit your research? Choose BMC and benefit from:

- fast, convenient online submission

- thorough peer review by experienced researchers in your field

- rapid publication on acceptance

- support for research data, including large and complex data types

- gold Open Access which fosters wider collaboration and increased citations

- maximum visibility for your research: over $100 \mathrm{M}$ website views per year

At $\mathrm{BMC}$, research is always in progress.

Learn more biomedcentral.com/submissions 